\title{
Preface
}

\section{Complex aortic arch surgery}

The first report of aortic arch repair by Borst in 1964 with hypothermic circulatory arrest (HCA) ushered in a new era of surgical therapy (1). Subsequently, Griepp reported a successful series of patients operated with HCA in 1975 and this new technique was increasingly used for a wide variety of aortic pathologies (2). Despite promising results, the time frame for advanced surgical techniques under HCA was limited by the risk of stroke and mortality which was improved with the addition of antegrade and retrograde perfusion techniques. Advancements of surgical techniques such as the "elephant trunk procedure" and simultaneous supra-aortic vessel reimplantation then became feasible.

Simultaneously, another treatment option using thoracic endovascular aortic repair (TEVAR) developed in the late 1990s. The "frozen elephant technique" combined both principles, the antegrade implantation of a stent graft. Further developments aimed at less invasive approaches combining endovascular aortic repair with surgical repair. Such hybrid procedures involved complex re-routing of the supra-aortic vessels and placement of a stent graft into arch or ascending aorta. Further developments in stent graft design have enabled endovascular replacement of the total aortic arch with minimal or even no surgical rerouting of the supra-aortic vessels.

In this issue, we explore 'complex aortic arch surgery' to review the myriad of approaches to the aortic arch. The decision process to perform traditional open, hybrid or total endovascular techniques is highly nuanced and new devices are continually providing surgeons with more options to address complex aortic arch pathology. Many of these options did not exist even 5 years ago. The innovation of branched arch grafts has disrupted the established open versus hybrid debate and these devices will continue to evolve and improve. Our aim is to provide an up to date evaluation of the full range of open, hybrid and endovascular procedures by the leaders and innovators in the field.

In this era of rapid technological progress, this issue is opened with a provocative and sanguine lecture by Professor Bachet highlighting the great successes of open arch surgery and potential advantages over rapidly advancing endovascular techniques. Prof. Svensson then provides a systematic approach to optimizing neurologic outcomes and achieving ideal clinical results. In the subsequent articles, current comparative data is reviewed, detailed technical aspects of the different surgical techniques are presented by true masters of the art of open and endovascular aortic surgery, and a strong emphasis is placed on neuroprotective strategies as stroke prevention remains a major concern in all open, hybrid and endovascular techniques in the arch. We hope this issue will help inform decisions regarding how to address complex aortic arch disease and encourage continued cross-specialty collaboration.

We express tremendous thanks to all of the contributors for their time and dedication to further the field of aortic surgery. We are very grateful to Professor Tristan Yan for the honorable invitation to serve as Guest Editors and to Ms. Kylie Cunningham and the ACS Editorial Office for all their efforts in assembling this compendium.

\section{Acknowledgements}

None.

\section{References}

1. Borst HG, Schaudig A, Rudolph W. Arteriovenous fistula of the aortic arch: repair during deep hypothermia and circulatory arrest. J Thorac Cardiovasc Surg 1964;48:443-7.

2. Griepp RB, Stinson EB, Hollingsworth JF, et al. Prosthetic replacement of the aortic arch. J Thorac Cardiovasc Surg 1975;70:1051-63.

Nimesh D. Desai, MD, PhD

Co-Director, Thoracic Aortic Surgery Program, Associate Professor of Surgery, Hospital of the University of Pennsylvania, Philadelphia, USA.

(Email: nimesh.desai@uphs.upenn.edu)

Eric E. Roselli, MD

Chief, Adult Cardiac Surgery, Surgical Director, Aorta Center, Director, HVI Condition Centers, Heart and Vascular Institute, Cleveland Clinic, Cleveland, USA.

(Email: roselle@ccf.org)

doi: 10.21037 /acs.2018.05.10

Conflicts of Interest: The authors have no conflicts of interest to declare.

View this article at: http://dx.doi.org/10.21037/acs.2018.05.10 